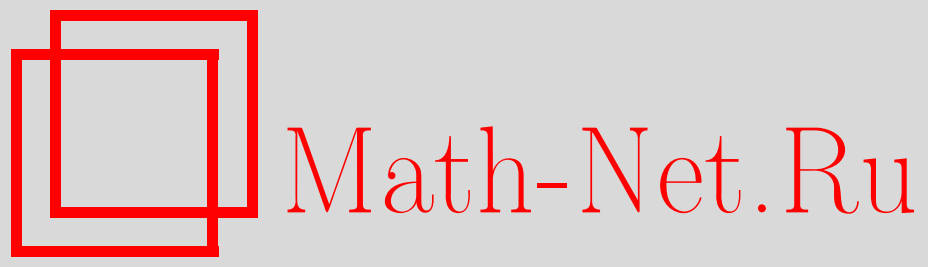

A. Kagan, M. Konikov, The structure of the UMVUEs from categorical data, Теория вероятн. и ее примен., 2005, том 50, выпуск 3, 597-604

DOI: https://doi.org/10.4213/tvp100

Использование Общероссийского математического портала Math-Net.Ru подразумевает, что вы прочитали и согласны с пользовательским соглашением

http: //www . mathnet.ru/rus/agreement

Параметры загрузки:

IP : 107.22 .136 .117

26 апреля 2023 г., 15:55:53

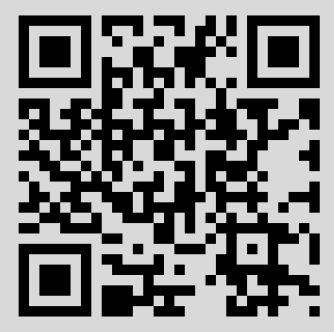


3. Balder E. J. New fundamentals of Young measure convergence. - Calculus of Variations and Optimal Control. Ed. by A. Ioffe, S. Reich, and I. Shafrir. Boca Raton: Chapman\&Hall/CRC Press, 2000, p. 24-48.

4. Blackwell D. On a theorem of Lyapunov. - Ann. Math. Statist., 1951, v. 22, p. 112114 .

5. Dvoretzky A., Wald A., Wolfowitz J. Elimination of randomization in certain problems of statistics and of the theory of games. - Proc. Natl. Acad. Sci. USA, 1950, v. 36, p. $256-260$.

6. Dvoretzky A., Wald A., Wolfowitz J. Elimination of randomization in certain statistical decision procedures and zero-sum two-person games. - Ann. Math. Statist., 1951, v. 22 , p. $1-21$.

7. Файнберг $E$. A. Нерандомизированные марковские и полумарковские стратегии в динамическом программировании. - Теория вероятн. и ее примен., 1982, т. 27, B. 1 , c. $109-119$.

8. Feinberg E. A. On measurability and representation of strategic measures in Markov decision processes. - Statistics, Probability and Game Theory. Papers in Honor of David Blackwell. Ed. by T. S. Ferguson, L. S. Shapley, and J. B. MacQueen. Hayward: Inst. Math. Statist., 1996, p. 29-43. (IMS Lecture Notes Monogr. Ser., v. 30.)

9. Feinberg E. A., Piunovskiy A. B. Nonatomic total rewards Markov decision processes with multiple criteria. - J. Math. Anal. Appl., 2002, v. 273, № 1, p. 93-111.

10. Ferguson T.S. Mathematical Statistics: A Decision Theoretic Approach. New YorkLondon: Academic Press, 1967, 396 p.

11. Пиуновский А.Б. Оптимальное управление случайными последовательностями в задачах с ограничениями. М: Научная книга, 1996.

Поступила в редакцию

1.VI.2005

(C) 2005 г. KAGAN A.*, KONIKOV M. $^{* *}$

\section{THE STRUCTURE OF THE UMVUES FROM CATEGORICAL DATA}

Пусть наблюдаемая случайная величина $X$ принимает конечное число значений с вероятностями $p_{1}(\theta), \ldots, p_{N}(\theta)$, зависящими от абстрактного параметра $\theta \in \Theta$. Доказывается, что статистика является несмещенной оценкой с равномерно минимальной дисперсией (UMVUE) тогда и только тогда, когда она измерима относительно подалгебры конечной алгебры, порожденной $X$. В общем случае эта подалгебра меньше, чем минимальная достаточная подалгебра для $\theta$, и описывается в явном виде. Она связана со специальным разбиением конечного множества элементов абстрактного линейного пространства.

Ключевые слова и фразы: оценка, линейное пространство, подалгебра, достаточность.

1. Introduction. Let $\mathscr{P}=\left\{\mathbf{P}_{\theta}, \theta \in \Theta\right\}$ be a family of probability distributions of a random element $X$ taking values in a measurable space $(\mathscr{X}, \mathscr{A})$. The structure of uniformly minimum variance unbiased estimators (UMVUEs) of parameter functions has interested statisticians since long ago. Due to the Rao-Blackwell theorem, a UMVUE is a function of the minimal sufficient statistic when the latter exists, in particular, for

* Department of Mathematics, University of Maryland, College Park, MD 20742; e-mail: amk@math.umd.edu

** Bloomberg LP, 499 Park Ave., New York, NY 10022, USA. 
dominated families $\mathscr{P}$. If the minimal sufficient statistic is complete, the class of UMVUEs coincides with the class of statistics with finite second moment that are functions of the minimal sufficient statistic (see, e.g., [6]).

Note in passing that when it exists, the minimal sufficient statistic is unique and a complete sufficient statistic is minimal.

We call a parameter function $\gamma(\theta), \gamma: \Theta \rightarrow \mathbf{R}$, estimable, if there exists a statistic $g(X)$, $g: \mathscr{X} \rightarrow \mathbf{R}$, such that

$$
\mathbf{E}_{\theta} g(X)=\gamma(\theta), \quad \mathbf{D}_{\theta} g(X)<\infty, \quad \theta \in \Theta .
$$

In [1] it is proved that if every estimable function possesses a UMVUE, there exists a minimal sufficient statistic for $\theta$. In a general case, some estimable parameter functions possess UMVUEs while the others do not.

Let $\chi(X)$ be an unbiased estimator of zero with finite variance, i.e.,

$$
\mathbf{E}_{\theta} \chi(X)=0, \quad \mathbf{D}_{\theta} \chi(X)<\infty, \quad \theta \in \Theta .
$$

The following well-known lemma (see, e.g., [5, Lemma 7.2.1]) gives a necessary and sufficient condition for a statistic $g(X)$ to be a UMVUE.

Lemma 1. A statistic $g(X)$ with finite second moment is a UMVUE if and only if it is uncorrelated with any unbiased estimator of zero $\chi(X)$, i.e.,

$$
\operatorname{cov}(g, \chi)=\mathbf{E}_{\theta}\{g(X) \chi(X)\}=0, \quad \theta \in \Theta .
$$

Plainly, if $g$ is a UMVUE, it is the UMVUE of the parameter function

$$
\gamma(\theta)=\mathbf{E}_{\theta} g(X) .
$$

An important observation was made in [8] (for a related result see [5, Theorem 7.2.1]). Suppose that $g(X)$ is a UMVUE. By Lemma $1,(1)$ holds for any unbiased estimator of zero $\chi(X)$ so that $g \chi$ is also an unbiased estimator of zero and, thus,

$$
\operatorname{cov}\left(g^{2}, \chi\right)=\mathbf{E}_{\theta}\left\{(g(X))^{2} \chi(X)\right\}=0, \quad \theta \in \Theta .
$$

Had this reasoning been flawless, one could have concluded that if $g$ is a UMVUE, then the statistics $1, g, g^{2}, \ldots$ with finite second moment are UMVUEs as well and, similarly, if $g_{1}, g_{2}$ are UMVUEs so is $g_{1} g_{2}$, etc. In other words, the class of UMVUEs would have been a ring.

The problem is that though $\mathbf{E}_{\theta} g^{2}<\infty$ and $\mathbf{E}_{\theta} \chi^{2}<\infty, \mathbf{E}_{\theta}(g \chi)^{2}$ may be infinite. In [1] an elegant example of a family $\mathscr{P}$ is constructed when the class of UMVUEs is not a ring.

However, in case of categorical data, i.e., when an observation $X$ takes finitely many values that, without loss in generality, may be taken as $1, \ldots, N$, all the statistics have finite moments of all orders and the class of UMVUEs is a ring.

Let

$$
\mathbf{P}_{\theta}\{X=i\}=p_{i}(\theta), \quad i \in I=\{1, \ldots, N\}, \quad \theta \in \Theta .
$$

Plainly, a function $\gamma(\theta)$ is estimable if and only if it is a linear combination of the probabilities,

$$
\gamma(\theta)=\sum_{1}^{N} c_{i} p_{i}(\theta) .
$$

In Section 3 it is proved that there exists a partition

$$
I=I_{1} \cup \cdots \cup I_{k}
$$

determined by the probabilities (2) such that $g(X)$ is a UMVUE if and only if it is constant on the elements of partition (4), i.e.,

$$
g(x)=g_{i}, \quad x \in I_{i}, \quad i=1, \ldots, k .
$$

An estimable parameter function $\gamma(\theta)$ possesses a UMVUE if and only if the coefficients in (3) are constant on the elements of (4),

$$
c_{j}=c_{i}^{*}, \quad j \in I_{i}, \quad i=1, \ldots, k .
$$


Partition (4), in general, is different from the minimal sufficient partition

$$
I=I_{1}^{\prime} \cup \cdots \cup I_{l}^{\prime}
$$

If one treats functions on $\Theta$ as elements of a linear vector space and sets

$$
p_{j}(\theta)=v_{j}, \quad j=1, \ldots, N
$$

then $i_{1}, i_{2}$ belong to the same element of partition (5) if and only if $v_{i_{1}} \| v_{i_{2}}$. In other words, if $S(X)$ is the minimal sufficient statistic for $\theta$, then

$$
S\left(i_{1}\right)=S\left(i_{2}\right) \Longleftrightarrow p_{i_{1}}(\theta)=c\left(i_{1}, i_{2}\right) p_{i_{2}}(\theta), \quad \theta \in \Theta,
$$

for a constant (in $\theta) c\left(i_{1}, i_{2}\right)$.

Each element of partition (4) is the union of elements of partition (5).

Looking at $p_{j}(\theta)$ as vectors translates the problem of describing the UMVUEs into a geometric problem in a finite dimensional linear space that may be of an interest in its own. Geometric lemmas are presented in Section 2.

Here we only illustrate the difference between partitions (4) and (5) with examples.

$\mathrm{Ex}$ a $\mathrm{m} \mathrm{ple} \mathrm{1.} \mathrm{Let}$

$$
p_{1}(\theta)=\theta, \quad p_{2}(\theta)=\theta^{2}, \quad p_{3}(\theta)=1-\theta-\theta^{2}, \quad \Theta=\left(0, \frac{1}{2}\right)
$$

Both partitions are $\{1\} \cup\{2\} \cup\{3\}$. The minimal sufficient statistic $S(X)=X$ is trivial and complete and all estimable functions (i.e., all quadratic polynomials in $\theta$ ) possess UMVUEs.

Exam ple 2. Let

$$
p_{1}(\theta)=\theta, \quad p_{2}(\theta)=\theta^{2}, \quad p_{3}(\theta)=\theta+\theta^{2}, \quad p_{4}(\theta)=1-2\left(\theta+\theta^{2}\right), \quad \Theta=\left(0, \frac{1}{4}\right) .
$$

The minimal sufficient statistic $S(X)$ is still trivial but plainly is incomplete since for any statistic $\chi(X)$ with

$$
\chi(1)=\chi(2)=-\chi(3), \quad \chi(4)=0 ;
$$

we have $\mathbf{E}_{\theta} \chi(X)=0, \theta \in \Theta$. Partition (4) is $\{1,2,3\} \cup\{4\}$ (see Section 3 for the proof) and the estimable functions possessing UMVUEs are of the form $a+b\left(\theta+\theta^{2}\right), a, b \in \mathbf{R}$, while, as in Example 1, all quadratic polynomials are estimable. A UMVUE satisfies the condition

$$
g(1)=g(2)=g(3)
$$

The structure of UMVUEs is of interest for foundations of statistics, at the very least. General results for incomplete exponential families were obtained in [3], [4] (see also [7]). An interesting general result is due [2]. Namely, let $\mathscr{P}=\left\{\mathbf{P}_{\theta, \xi},(\theta, \xi) \in \Theta \times \Xi\right\}$ be a family of distributions on a measurable space parameterized by a «bivariate» parameter $(\theta, \xi)$, $\Theta, \Xi$ being arbitrary sets. If for each fixed $\xi$, a statistic $T$ is complete sufficient for $\theta$ (but $T$ is not assumed sufficient for $(\theta, \xi))$, then any statistic $g(T)$ with finite second moment is a UMVUE.

The description of the class of all UMVUEs from categorical data presented in the next sections is not related to sufficiency and is based on properties of finite dimensional linear spaces. The structure of the class is different from the known structures.

2. Geometric lemmas. Let $L$ be an abstract linear space with zero element 0. Subsets $U_{1}, \ldots, U_{n}$ of $L$ are called linearly independent if so are the subspaces $\operatorname{span}\left(U_{1}\right), \ldots$, $\operatorname{span}\left(U_{n}\right)$, i.e., the relation

$$
u_{1}+\cdots+u_{n}=\mathbf{0}, \quad u_{i} \in \operatorname{span}\left(U_{i}\right), \quad i=1, \ldots, n,
$$

implies

$$
u_{1}=\cdots=u_{n}=\mathbf{0} .
$$

The following lemma is a key to the structure of the class of UMVUEs from categorical data. 
Lemma 2. Let $V=\left\{v_{1}, \ldots, v_{N}\right\}$ be a finite set of elements of $L$; it is assumed that $\mathbf{0} \notin V$. There exists a partition

$$
V=V_{1} \cup \cdots \cup V_{k}
$$

such that (i) $V_{1}, \ldots, V_{k}$ are linearly independent, (ii) (6) is irreducible, i.e., if for some $j \in\{1, \ldots, k\}$

$$
V_{j}=V_{j 1} \cup V_{j 2}, \quad V_{j 1} \cap V_{j 2}=\varnothing
$$

and $V_{1}, \ldots, V_{j-1}, V_{j 1}, V_{j 2}, V_{j+1}, \ldots, V_{k}$ are linearly independent, then either $V_{j 1}=V_{j}$ or $V_{j 2}=V_{j}$.

Such a partition is unique up to the order in (6) and within $V_{1}, \ldots, V_{k}$. that

$\mathrm{P}$ r o o f. We proceed by induction. For $N=1$ Lemma 2 is trivially true. Suppose

$$
V^{\prime}=V_{1}^{\prime} \cup \cdots \cup V_{m}^{\prime}
$$

is the claimed partition of the set $V^{\prime}=\left\{v_{1}, \ldots, v_{N-1}\right\}$. If $V_{1}^{\prime}, \ldots, V_{m}^{\prime},\left\{v_{N}\right\}$ are linearly independent, the partition

$$
V=V_{1}^{\prime} \cup \cdots \cup V_{m}^{\prime} \cup V_{m+1}^{\prime}, \quad V_{m+1}^{\prime}=\left\{v_{N}\right\}
$$

satisfies (i) and (ii).

Suppose now that $V_{1}^{\prime}, \ldots, V_{m}^{\prime},\left\{v_{N}\right\}$ are linearly dependent. Due to the assumed linear independence of $V_{1}^{\prime}, \ldots, V_{m}^{\prime}$, there is exactly one collection $\left(V_{i_{1}}^{\prime}, \ldots, V_{i_{l}}^{\prime}\right), i_{1}, \ldots, i_{l} \in$ $\{1, \ldots, m\}$, such that $v_{N} \in \operatorname{span}\left(V_{i_{1}}^{\prime}, \ldots, V_{i_{l}}^{\prime}\right)$ and

$$
v_{N}=v_{i_{1}}+\cdots+v_{i_{l}} \quad \text { with } \quad v_{i_{1}} \in V_{i_{1}}, \ldots, v_{i_{l}} \in V_{i_{l}}, \quad v_{i_{1}} \neq \mathbf{0}, \ldots, v_{i_{l}} \neq \mathbf{0} .
$$

Without loss in generality, one may assume

$$
V_{i_{1}}^{\prime}=V_{1}^{\prime}, \ldots, V_{i_{l}}^{\prime}=V_{l}^{\prime}, \quad l \leqslant m .
$$

The partition

$$
V=V_{1} \cup \cdots \cup V_{k}
$$

with $k=m-l+1$ and

$$
V_{1}=V_{1}^{\prime} \bigcup \cdots \cup V_{l}^{\prime} \bigcup\left\{v_{N}\right\}, \quad V_{2}=V_{l+1}^{\prime}, \ldots, V_{k}=V_{m}^{\prime}
$$

satisfies (i) and (ii). Indeed, linear independence of $V_{1}, \ldots, V_{k}$ follows from linear independence of $V_{1}^{\prime}, \ldots, V_{m}^{\prime}$ and the fact that $v_{N} \in \operatorname{span}\left(V_{1}^{\prime}, \ldots, V_{l}^{\prime}\right)$.

Suppose now that

$$
V_{1}=V_{11} \bigcup V_{12}, \quad V_{11} \bigcap V_{12}=\varnothing .
$$

If $v_{N} \in \operatorname{span}\left(V_{11}\right)$ and $V_{12} \neq \varnothing$ or $v_{N} \in \operatorname{span}\left(V_{12}\right)$ and $V_{11} \neq \varnothing$, either pair of conditions contradicts (7).

To prove the uniqueness, assume that, besides (6), there is another partition with properties (i) and (ii) of Lemma 2,

$$
V=\widetilde{V}_{1} \cup \cdots \cup \widetilde{V}_{n}
$$

for some $n$. Take the intersections

$$
V_{i 1}=V_{i} \cap \widetilde{V}_{1}, \ldots, V_{i n}=V_{i} \cap \widetilde{V}_{n}, \quad i=1, \ldots, k .
$$

They are linearly independent since (8) satisfies (i). But this contradicts irreducibility of (6) unless only one of the sets (9) is nonempty. The same arguments prove that only one of the intersections

$$
\widetilde{V}_{j 1}=\widetilde{V}_{j} \cap V_{1}, \ldots, \widetilde{V}_{j k}=\widetilde{V}_{j} \cap V_{k}, \quad j=1, \ldots, n,
$$

is nonempty. This proves that for the partitions (6) and (8), $k=n$ and each $\widetilde{V}_{j}$ is one of $V_{i}$. Lemma 2 is proved.

Plainly, collinear vectors belong to the same component of partition (6). 
Ex a m p le 3. Let $u_{1}, u_{2}, u_{3}$ be linearly independent elements of $L$ and $V=$ $\left\{v_{1}, v_{2}, v_{3}, v_{4}\right\}$, where

$$
v_{1}=u_{1}, \quad v_{2}=u_{2}, \quad v_{3}=u_{2}+u_{3}, \quad v_{4}=u_{1}+u_{2} .
$$

Then $V^{\prime}=V_{1}^{\prime} \cup V_{2}^{\prime} \cup V_{3}^{\prime}$ with $V_{1}^{\prime}=\left\{v_{1}\right\}, V_{2}^{\prime}=\left\{v_{2}\right\}, V_{3}^{\prime}=\left\{v_{3}\right\}, v_{4} \in \operatorname{span}\left(V_{1}^{\prime}, V_{2}^{\prime}\right)$ and $V=V_{1} \cup V_{2}$ with $V_{1}=V_{1}^{\prime} \cup V_{2}^{\prime} \cup\left\{v_{4}\right\}, V_{2}=V_{3}^{\prime}$.

$\mathrm{E} \times \mathrm{a} \mathrm{m} \mathrm{p} \mathrm{le} \mathrm{4.} \mathrm{Add} v_{5}=u_{3}$ and let $V=\left\{v_{1}, v_{2}, v_{3}, v_{4}, v_{5}\right\}$. Then

$$
V^{\prime}=V_{1}^{\prime} \cup V_{2}^{\prime} \quad \text { with } \quad V_{1}^{\prime}=\left\{v_{1}, v_{2}, v_{4}\right\}, V_{2}^{\prime}=\left\{v_{3}\right\}
$$

and $v_{5} \in \operatorname{span}\left(V_{1}^{\prime}, V_{2}^{\prime}\right)$. In this case, the partition is trivial, $V=V_{1}$.

The partition (6) generates a partition of the set $I=\{1, \ldots, N\}$,

$$
I=I_{1} \bigcup \cdots \cup I_{k}, \quad I_{j}=\left\{i \in I: v_{i} \in V_{j}\right\}, \quad j=1, \ldots, k .
$$

A linear combination $\sum_{i=1}^{N} c_{i} v_{i}$ is called a contrast if

$$
\sum_{i \in I_{1}} c_{i} v_{i}=\mathbf{0}
$$

A contrast is called decomposable if there is a partition $I=I^{\prime} \cup I^{\prime \prime}$ such that $\sum_{i \in I^{\prime}} c_{i} v_{i}$ and $\sum_{i \in I^{\prime \prime}} c_{i} v_{i}$ are nontrivial contrasts (i.e., not all the coefficients equal zero) and indecomposable otherwise.

Corollary 1. If $\sum_{1}^{N} c_{i} v_{i}$ is a contrast and

$$
\sum_{1}^{N} c_{i} v_{i}=\sum_{i \in I_{1}} c_{i} v_{i}+\cdots+\sum_{i \in I_{k}} c_{i} v_{i},
$$

then

$$
\sum_{i \in I_{1}} c_{i} v_{i}, \ldots, \sum_{i \in I_{k}} c_{i} v_{i}
$$

are also contrasts.

P r o o f. Indeed, since

$$
\sum_{i \in I_{1}} c_{i} v_{i}=u_{1} \in \operatorname{span}\left(V_{1}\right), \ldots, \sum_{i \in I_{k}} c_{i} v_{i}=u_{k} \in \operatorname{span}\left(V_{k}\right)
$$

the relation $u_{1}+\cdots+u_{k}=\mathbf{0}$ implies $u_{1}=\cdots=u_{k}=\mathbf{0}$.

Turn now to contrasts (12).

Lemma 3. Suppose that $V_{1}$ contains at least two vectors. For any two vectors $v_{i}$, $v_{j} \in V_{1}$, there exists an indecomposable contrast $\sum_{i \in I_{1}} c_{i} v_{i}$ with $c_{i} c_{j} \neq 0$.

$\mathrm{P}$ r o of. Re-enumerating the vectors $v_{1}, \ldots, v_{N}$, if necessary, one may assume that $V_{1}=\left(v_{1}, v_{2}, \ldots, v_{m+1}\right)$. It suffices to prove Lemma 3 for $v_{1}, v_{2}$.

We shall proceed by induction in $\left|I_{1}\right|$, the number of elements in $V_{1}$. For $V_{1}=\left(v_{1}, v_{2}\right)$, the vectors $v_{1}$ and $v_{2}$ are linearly dependent (otherwise they would have belonged to different components of the partition) and neither is $\mathbf{0}$. Hence $c_{1} v_{1}+c_{2} v_{2}=\mathbf{0}$ for some $c_{1}, c_{2}$ with $c_{1} c_{2} \neq 0$.

Now assume that Lemma 3 holds for $\left|I_{1}\right| \leqslant m$ and prove it for $\left|I_{1}\right|=m+1$. If the set $\left(v_{1}, v_{2}, \ldots, v_{m}\right)$ is irreducible, the claim holds true due to the induction assumption.

Suppose now that $\left(v_{1}, v_{2}, \ldots, v_{m}\right)$ is reducible (though $V_{1}$ is irreducible). By virtue of Lemma 2 , there is a partition

$$
V_{1}=V_{11} \cup V_{12} \bigcup \cdots \cup V_{1 l}
$$

similar to (6). If $v_{1}, v_{2}$ belong to the same component of the partition (13), the claim of Lemma 3 again holds due to the induction assumption.

Consider the case when $v_{1}, v_{2}$ belong to different components of (13), $v_{1} \in V_{11}, v_{2} \in V_{12}$, say. There is no loss in generality in assuming that a basis of $\operatorname{span}\left(V_{1 i}\right), i=1, \ldots, l$, consists of elements of $V_{1 i}$,

$$
v_{1 i}, v_{2 i}, \ldots, v_{m_{i} i}, \quad v_{11}=v_{1}, \quad v_{21}=v_{2} .
$$


Since

$$
v_{11}, \ldots, v_{1 m_{1}} ; v_{21}, \ldots, v_{2 m_{2}} ; \ldots ; v_{l 1}, \ldots, v_{l m_{l}}
$$

is a basis of $\operatorname{span}\left(V_{11}, V_{12}, \ldots, V_{1 l}\right)$ and $V_{1}$ is irreducible, there exists a unique representation

$$
v_{m+1}=\sum_{i=1}^{m_{1}} c_{1 i} v_{1 i}+\sum_{i=1}^{m_{2}} c_{2 i} v_{2 i}+\cdots+\sum_{i=1}^{m_{l}} c_{l i} v_{l i} .
$$

One has $c_{11} c_{21} \neq 0$. Indeed, were $c_{11}=0$ it would imply that $v_{1}=v_{11}$ is linearly independent of $v_{2}, \ldots, v_{m+1}$ contradicting irreducibility of $V_{1}$. Similarly, $c_{21}=0$ implies the linear independence of $v_{2}$ and $v_{1}, v_{3}, \ldots, v_{m+1}$. Thus,

$$
\sum_{i=1}^{m_{1}} c_{1 i} v_{1 i}+\sum_{i=1}^{m_{2}} c_{2 i} v_{2 i}+\cdots+\sum_{i=1}^{m_{l}} c_{l i} v_{l i}-v_{m+1}
$$

is the required contrast. It is indecomposable due to the uniqueness of the representation (14). Lemma 3 is proved.

Let $c=\left(c_{1}, \ldots, c_{N}\right)^{T}, g=(g(1), \ldots, g(N))^{T}$ be vectors in $\mathbf{R}^{N}$. The Hadamard product $h=g \odot c$ of $g$ and $c$ is the vector with components $h_{1}=g(1) c_{1}, \ldots, h_{N}=g(N) c_{N}$.

Lemma 4. Suppose $g$ is such that for any contrast $\sum_{1}^{N} c_{i} v_{i}, \sum_{1}^{N} h_{i} v_{i}$ is also a contrast. Then $g$ is constant on each $I_{l}, l=1, \ldots, k$, i.e.,

$$
g(x)=g_{1}, x \in I_{1}, \ldots, g(x)=g_{k}, x \in I_{k} .
$$

$\mathrm{P} \mathrm{r}$ o o f. The claim of Lemma 4 is nontrivial only for the components of (10) containing at least two elements. Assuming that $I_{1}$ is such and $v_{1}, v_{2} \in V_{1}$, we shall prove that $g(1)=g(2)$. Dividing $g(x)$ by $\max \left\{|g(x)|, x \in I_{1}\right\}$, one may assume that $|g(x)| \leqslant 1$, $x \in I_{1}$. Let $\sum_{i \in I_{1}} c_{i} v_{i}$ be an indecomposable contrast with $c_{1} c_{2} \neq 0$. Such a contrast exists due to Lemma 3.

Using the condition of Lemma 4 repeatedly, we get that the linear combinations

$$
\sum_{i \in I_{1}} g(i) c_{i} v_{i}, \sum_{i \in I_{1}}[g(i)]^{2} c_{i} v_{i}, \ldots, \sum_{i \in I_{1}}[g(i)]^{n} c_{i} v_{i}
$$

are all contrasts.

Let $I_{1}=I_{1}^{\prime} \cup I_{1}^{\prime \prime}$, where

$$
I_{1}^{\prime}=\left\{i \in I_{1}:|g(i)|=1\right\}, \quad I_{1}^{\prime \prime}=\left\{i \in I_{1}:|g(i)|<1\right\} .
$$

Letting $n=2,4,6, \ldots \rightarrow \infty$ in (16) leads to that $\sum_{i \in I_{1}^{\prime}} c_{i} v_{i}$ is a contrast which contradicts indecomposability of $\sum_{i \in I_{1}}$ unless $I_{1}^{\prime \prime}=\varnothing$.

Let now $I_{1}^{+}=\left\{i \in I_{1}: g(i)=+1\right\}, I_{1}^{-}=\left\{i \in I_{1}: g(i)=-1\right\}$. Since

$$
\sum_{i \in I_{1}} g(i) c_{i} v_{i}=\sum_{i \in I_{1}^{+}} c_{i} v_{i}-\sum_{i \in I_{1}^{-}} c_{i} v_{i}
$$

we have $\sum_{i \in I_{1}^{+}} c_{i} v_{i}=\sum_{i \in I_{1}^{-}} c_{i} v_{i}$ and thus $\sum_{i \in I_{1}^{+}} c_{i} v_{i}$ and $\sum_{i \in I_{1}^{-}} c_{i} v_{i}$ are contrasts again contradicting indecomposability of the contrast $\sum_{i \in I_{1}} c_{i} v_{i}$ unless one of the sets $I_{1}^{+}, I_{1}^{-}$is empty. Since $c_{1} c_{2} \neq 0$, we have $g(1)=g(2)$. Lemma 4 is proved.

3. Main result. Return to the statistical setup when an observation $X$ takes values $1, \ldots, N$ with probabilities (2).

Theorem 1. An estimable parameter function (3) has a UMVUE if and only if

$$
c_{i}=c_{j}^{*}, \quad i \in I_{j}, \quad j=1, \ldots, k,
$$

with the sets $I_{1}, \ldots, I_{k}$ defined in (10).

A statistic $g(X)$ is a UMVUE if and only if

$$
g(x)=g_{j}^{*}, \quad x \in I_{j}, \quad j=1, \ldots, k .
$$


P r o of. Let $\chi(X)$ be an unbiased estimator of zero,

$$
\mathbf{E}_{\theta} \chi(X)=\sum_{1}^{N} \chi(i) p_{i}(\theta)=0, \quad \theta \in \Theta
$$

On looking at $p_{j}(\theta)$ as an element $v_{j}$ of the linear space $L$ of functions on $\Theta$, the unbiased estimator of zero from (19) becomes a contrast $\sum_{1}^{N} c_{i} v_{i}$ with $c_{i}=\chi(i)$.

By Lemma 1, a statistic $g(X)$ is a UMVUE if for any unbiased estimator of zero $\chi(X)$,

$$
\mathbf{E}_{\theta}\{g(X) \chi(X)\}=\sum_{1}^{N} g(i) \chi(i) p_{i}(\theta)=0, \quad \theta \in \Theta .
$$

In terms of $L,(20)$ means that $g=(g(1), \ldots, g(N))^{T} \in \mathbf{R}^{N}$ is such a vector that for any contrast $\sum_{1}^{N} c_{i} v_{i}, \sum_{1}^{N} h_{i} v_{i}$ with $h_{i}=g(i) c_{i}, i=1, \ldots, N$, is also a contrast.

By Lemma 4, such a $g$ must be constant on each $I_{j}$ thus proving (18).

Conversely, suppose that (18) holds. By virtue of Corollary $1, \sum_{i \in I_{j}} c_{i} v_{i}$ is a contrast for $j=1, \ldots, k$, i.e.,

$$
\sum_{i \in I_{j}} \chi(i) p_{i}(\theta)=0, \quad \theta \in \Theta, \quad j=1, \ldots, k,
$$

and thus

$$
\mathbf{E}_{\theta}\{g(X) \chi(X)\}=\sum_{1}^{N} g(i) \chi(i) p_{i}(\theta)=\sum_{j=1}^{k} g_{j}^{*}\left[\sum_{i \in I_{j}} \chi(i) p_{i}(\theta)\right]=0, \quad \theta \in \Theta .
$$

This proves the second claim of Theorem 1 .

For $g(X)$ satisfying (18),

$$
\mathbf{E}_{\theta} g(X)=\gamma(\theta)=\sum_{j=1}^{k} g_{j}^{*}\left[\sum_{i \in I_{I}} p_{i}(\theta)\right]
$$

proving (17). Theorem 1 is proved.

Corollary 2. If for some $i \in I$,

$$
p_{i}(\theta)=p_{i}>0
$$

does not depend on $\theta$, any UMVUE is a constant.

P r o o f. Since $p_{1}(\theta)+\cdots+p_{N}(\theta)=1=\lambda p_{i}$ for some $\lambda>0$, partition (6) is trivial, $V=V_{1}$, and so is partition (8), $I=I_{1}$.

$\mathrm{Ex} \mathrm{a} \mathrm{m} \mathrm{p} \mathrm{l} \mathrm{e} \mathrm{5.} \mathrm{A} \mathrm{pair} \mathrm{of} \mathrm{coins,} \mathrm{one} \mathrm{fair,} \mathrm{the} \mathrm{other} \mathrm{with} \mathrm{probability} \theta$ of falling head as a parameter is tossed independently $n$ times. In the $i$-th tossing, only the number of heads $X_{i}$ is recorded (but not which coin falls what). An observation $X$ is the vector $\left(X_{1}, \ldots, X_{n}\right)$. There is no UMVUE of $\theta$ from $X$ (neither of any other nonconstant parameter function) since

$$
\mathbf{P}_{\theta}\{X=(1, \ldots, 1)\}=2^{-n}
$$

does not depend on $\theta$.

Note in passing that Corollary 2 holds in a general setup (not necessarily of categorical data). Namely, let $\mathscr{P}=\left\{\mathbf{P}_{\theta}, \theta \in \Theta\right\}$ be a dominated family of distributions of a random element $X$ taking values in a measurable space $(\mathscr{X}, \mathscr{A})$ with $d \mathbf{P}_{\theta} / d \mu=p(x ; \theta)$ for some measure $\mu$.

If $p(x ; \theta)=p(x)>0, x \in A$ and $\mu(A)>0$, then any UMVUE from $X$ is a constant.

Let us look at Examples 1, 2 from Section 1.

In Example 1 the vectors $v_{1}=\theta, v_{2}=\theta^{2}, v_{3}=1-\left(\theta+\theta^{2}\right), \theta \in\left(0, \frac{1}{2}\right)$, are linearly independent and (6) holds with $V_{1}=\left\{v_{1}\right\}, V_{2}=\left\{v_{2}\right\}, V_{3}=\left\{v_{3}\right\}$. Correspondingly, $I_{1}=\{1\}, I_{2}=\{2\}, I_{3}=\{3\}$ and any statistic is a UMVUE.

In Example 2 the vectors are $v_{1}=\theta, v_{2}=\theta^{2}, v_{3}=\theta+\theta^{2}$, and $v_{4}=1-2\left(\theta+\theta^{2}\right)$, $\theta \in\left(0, \frac{1}{4}\right)$, so that $v_{1}, v_{2}, v_{3}$ are linearly dependent (but any two of them are linearly 
independent) and $v_{4}$ is linearly independent of $\operatorname{span}\left(v_{1}, v_{2}, v_{3}\right)$. Partition (6) holds with $V_{1}=\left\{v_{1}, v_{2}, v_{3}\right\}, V_{2}=\left\{v_{4}\right\}$ and $I_{1}=\{1,2,3\}, I_{2}=\{4\}$. A statistic $g(X)$ is a UMVUE if and only if $g(1)=g(2)=g(3)$ and only the quadratic polynomials $a+b\left(\theta+\theta^{2}\right)$ possess UMVUEs.

$\mathrm{E} \times \mathrm{a} \mathrm{m} \mathrm{p}$ e 6 . Let $X$ be a binomial random variable $\operatorname{Bin}(n, \theta)$,

$$
\mathbf{P}_{\theta}\{X=j\}=p_{j}(\theta)=\left(\begin{array}{c}
n \\
j
\end{array}\right) \theta^{j}(1-\theta)^{n-j}, \quad j=0,1, \ldots, n .
$$

As is well known, $v_{0}=p_{0}(\theta), v_{1}=p_{1}(\theta), \ldots, v_{n}=p_{n}(\theta)$ are linearly independent so that (6) holds with

$$
V_{1}=\left\{v_{0}\right\}, \ldots, V_{n+1}=\left\{v_{n}\right\} .
$$

Any statistic $g(X)$ is a UMVUE and every polynomial of degree $\leqslant n$ in $\theta$ possesses a UMVUE.

Suppose now that $X$ is additively perturbed by an independent binary random variable $Y$ with a known

$$
\mathbf{P}\{Y=1\}=1-\mathbf{P}\{Y=0\}=\varepsilon
$$

and what is observed is

$$
\widetilde{X}=X+Y \text {. }
$$

The distribution of $\widetilde{X}, \mathbf{P}_{\theta}\{\widetilde{X}=j\}=\widetilde{p}_{j}(\theta), j=0,1, \ldots, n+1$, is

$$
\widetilde{p}_{0}(\theta)=(1-\varepsilon) p_{0}(\theta), \widetilde{p}_{1}(\theta)=\varepsilon p_{0}(\theta)+(1-\varepsilon) p_{1}(\theta), \ldots, \widetilde{p}_{n+1}(\theta)=\varepsilon p_{n}(\theta)
$$

and the vectors $\widetilde{v}_{j}, j=0,1, \ldots, n+1$, are linearly dependent $(n+2$ polynomials of degree $\leqslant n$ ) so that partition (6) of $\widetilde{V}=\left\{\widetilde{v}_{0}, \ldots, \widetilde{v}_{n+1}\right\}$ is trivial,

$$
\widetilde{V}=\widetilde{V}_{1}
$$

and only constants are UMVUEs from $\widetilde{X}$.

The last example is an illustration of a general principle that, as a rule, the optimal procedures are not robust with respect to perturbations of the model.

\section{REFERENCES}

1. Bahadur R. R. On unbiased estimates of uniformly minimum variance. - Sankhyā, 1957 , v. 18 , p. 211-224.

2. Bondesson L. On uniformly minimum variance unbiased estimation when no complete sufficient statistics exist. - Metrika, 1983, v. 30, № 1, p. 49-54.

3. Каган А.М., Линник Ю. В. Несмещенное оценивание для неполных экспоненциальных семейств. - Transactions of the Fourth Prague Conference on Information Theory, Statistical Decision Functions, Random Processes (Prague, 1965). Prague: Academia, 1967, p. 389-398.

4. Каган А.М., Паламодов В.П. Неполные экспоненциальные семейства и несмешенные оценки с наименьшей дисперсией. - Теория вероятн. и ее примен., 1967, т. 12 , в. 1 , c. $39-50$.

5. Каган А.М., Линник Ю. В., Рао С.Р. Характеризашионные задачи математической статистики. М.: Наука, 1972, 656 с.

6. Леман Э. Теория точечного оценивания. М.: Наука, 1991, 444 с.

7. Линник Ю. В. Статистические задачи с мешаюшими параметрами. - М.: Наука, 1966, 252 c.

8. Rao C. R. Some theorems on minimum variance estimation. - Sankhyā, 1952, v. 12, p. $27-42$. 